\title{
A Shocking Diagnosis
}

The approach to clinical conundrums by an expert clinician is revealed through the presentation of an actual patient's case in an approach typical of a morning report. Similarly to patient care, sequential pieces of information are provided to the clinician, who is unfamiliar with the case. The focus is on the thought processes of both the clinical team caring for the patient and the discussant.

This icon represents the patient's case. Each paragraph that follows represents the discussant's thoughts.

Anbazhagan Prabhakaran, MD ${ }^{1 *}$, Gurpreet Dhaliwal, MD², Christopher Robert-James Schilf, MD', George H. Caughey, MD², James Pile, MD ${ }^{4}$

${ }^{1}$ Medicine Institute, Cleveland Clinic, Cleveland, Ohio; ${ }^{2}$ Department of Medicine, University of California, San Francisco, California; ${ }^{3}$ Medical Service, San Francisco VA Medical Center, San Francisco, California; ${ }^{4}$ Department of Medicine, MetroHealth Medical Center, Case Western Reserve University, Cleveland, Ohio.

门 A 75-year-old man was brought by ambulance to the emergency department (ED) after the acute onset of palpitations, lightheadedness, and confusion. His medical history, provided by his wife, included osteoarthritis and remote cholecystectomy. He was not a smoker but drank 2 to 4 cans of beer daily. His medications were aspirin 162 $\mathrm{mg}$ daily and naproxen as needed. There was no history of bruising, diarrhea, melena, or bleeding.

Palpitations may represent an arrhythmia arising from an ischemic or alcoholic cardiomyopathy. Mental status changes usually have metabolic, infectious, structural (eg, hemorrhage, tumor), or toxic causes. Lightheadedness and confusion could occur with arrhythmia-associated cerebral hypoperfusion or a seizure. Daily alcohol use could cause confusion through acute intoxication, thiamine or $\mathrm{B}_{12}$ deficiency, repeated head trauma, or liver failure.

The patient's systolic blood pressure (BP) was $60 \mathrm{~mm}$ $\mathrm{Hg}$, heart rate (HR) was 120 beats per minute (bpm), and oral temperature was $98.4^{\circ} \mathrm{F}$. Rousing him was difficult. There were no localizing neurologic abnormalities, and the rest of the physical examination findings were normal. Point-of-care blood glucose level was $155 \mathrm{mg} / \mathrm{dL}$. Blood cultures were obtained and broad-spectrum antibiotics initiated. After fluid resuscitation, BP improved to $116 / 87 \mathrm{~mm}$ $\mathrm{Hg}$, HR fell to $105 \mathrm{bpm}$, and the patient became alert and oriented. He denied chest pain, fever, or diaphoresis.

The patient's improvement with intravenous (IV) fluids makes cardiogenic shock unlikely but does not exclude an

\footnotetext{
*Address for correspondence and reprint requests: Anbazhagan Prabhakaran, MD, Medicine Institute, M2 Annex, Cleveland Clinic, 9500 Euclid Ave, Cleveland, OH 44195; Telephone: 216-538-9022; Fax: 216-444-8530; E-mail: prabhak@ccf.org
}

Received: May 23, 2016; Revised: August 8, 2016; Accepted: August 20, 2016

2017 Society of Hospital Medicine DOI 10.12788/jhm.2690 underlying compensated cardiomyopathy that may be predisposing to arrhythmia. Hypotension, tachycardia, and somnolence may represent sepsis, but the near normalization of vital signs and mental status shortly after administration of IV fluids, the normal temperature, and the absence of localizing signs of infection favor withholding additional antibiotics. Other causes of hypotension are hypovolemia, medication effects, adrenal insufficiency, anaphylaxis, and autonomic insufficiency. There was no reported nausea, vomiting, diarrhea, bleeding, polyuria, or impaired oral intake to support hypovolemia, though the response to IV fluids suggests hypovolemia may still be playing a role.

White blood cell (WBC) count was $15,450 / \mu \mathrm{L}$ with a normal differential; hemoglobin level was $15.8 \mathrm{~g} / \mathrm{dL}$; and platelet count was $176,000 / \mu \mathrm{L}$. Electrolytes, liver function tests, cardiac enzymes, and urinalysis were normal. Electrocardiogram showed sinus tachycardia with premature atrial complexes and no ST-segment abnormalities. Radiograph of the chest and computed tomography scan of the head were normal. Echocardiogram showed moderate left ventricular hypertrophy with a normal ejection fraction and no valvular abnormalities. Exercise nuclear cardiac stress test was negative for ischemia. Blood cultures were sterile. The patient quickly became asymptomatic and remained so during his 3-day hospitalization. There were no arrhythmias on telemetry. The patient was discharged with follow-up scheduled with his primary care physician.

The nonlocalizing history and physical examination findings, normal chest radiograph and urinalysis, absence of fevers, negative blood cultures, and quick recovery make infection unlikely, despite the moderate leukocytosis. Conditions that present with acute and transient hypotension and altered mental status include arrhythmias, seizures, and reactions to drugs or toxins. Given the cardiac test results, a chronic cardiomyopathy seems unlikely, but arrhythmia is still possible. Continuous outpatient monitoring is required to assess the palpitations and the frequency of the premature atrial complexes. 
Two days after discharge, the patient suddenly became diaphoretic and lost consciousness while walking to the bathroom. He was taken to the ED, where his BP was 90/60 mm Hg and HR was 108 bpm. Family members reported that he had appeared flushed during the syncopal episode, showed no seizure activity, and been unconscious for 15 to 20 minutes. The patient denied chest pain, dyspnea, fever, bowel or bladder incontinence, focal weakness, slurred speech, visual changes, nausea or vomiting either before or after the episode. Physical examination revealed a tongue laceration and facial erythema; all other findings were normal. In the ED, there was an asymptomatic 7-beat run of nonsustained ventricular tachycardia, and the hypotension resolved after fluid resuscitation. The patient now reported 2 similar syncopal episodes in the past. The first occurred in a restaurant 6 years earlier, and the second occurred 3 years later, at which time he was hospitalized and no etiology was found.

The loss of consciousness is attributable to cerebral hypoperfusion. Hypotension has 3 principal categories: hypovolemic, cardiogenic, and distributive. With syncopal episodes recurring over several years, hypovolemia seems unlikely. Given the palpitations and ventricular tachycardia, it is reasonable to suspect a cardiogenic cause. Although his heart appears to be structurally normal on echocardiogram, genetic, electrophysiologic, or magnetic resonance imaging (MRI) testing will occasionally reveal an unsuspected substrate for arrhythmia.

The recurring yet self-limited nature, diaphoresis, flushing, and facial erythema suggest a non-sepsis distributive cause of hypotension. It is possible the patient is recurrently exposed to a toxin (eg, alcohol) that causes both flushing and dehydration. Flushing disorders include carcinoid syndrome, pheochromocytoma, drug reaction with eosinophilia and systemic symptoms (DRESS), and mastocytosis. Carcinoid syndrome is characterized by bronchospasm and diarrhea and, in some cases, right-sided valvulopathy, all of which are absent in this patient. Pheochromocytoma is associated with orthostasis, but patients typically are hypertensive at baseline. DRESS, which may arise from nonsteroidal anti-inflammatory drug (NSAID) or aspirin use, can cause facial erythema and swelling but is also characterized by liver, renal, and hematologic abnormalities, none of which was demonstrated. Furthermore, DRESS typically does not cause hypotension. Mastocytosis can manifest as isolated or recurrent anaphylaxis.

It is important to investigate antecedents of these syncopal episodes. If the earlier episodes were food-related-one occurred at a restaurant-then deglutition syncope (syncope precipitated by swallowing) should be considered. If an NSAID or aspirin was ingested before each episode, then medication hypersensitivity or mast cell degranulation (which can be triggered by these medications) should be further examined. Loss of consciousness lasting 20 minutes without causing any neurologic sequelae is unusual for most causes of recurrent syncope. This feature raises the possibility that a toxin or mediator might still be present in the patient's system.

Serial cardiac enzymes and electrocardiogram were normal. A tilt-table study was negative. The cortisol response to $\mathrm{ACTH}$ (cosyntropin) stimulation was normal. The level of serum tryptase, drawn 2 days after syncope, was $18.4 \mathrm{ng} / \mathrm{dL}$ (normal, $<11.5 \mathrm{ng} / \mathrm{dL}$ ). Computed tomography scan of chest and abdomen was negative for pulmonary embolism but showed a $1.4 \times 1.3-\mathrm{cm}$ hypervascular lesion in the tail of pancreas. The following neuroendocrine tests were within normal limits: serum and urine catecholamines; urine 5-hydroxyindoleacetic acid (5-HIAA); and serum chromogranin $A$, insulin, serotonin, vasoactive intestinal polypeptide (VIP), and somatostatin (Table 1). The patient remained asymptomatic during his hospital stay and was discharged home with appointments for cardiology follow-up and endoscopic ultrasound-guided biopsy of the pancreatic mass.

Pheochromocytoma is unlikely with normal serum and urine catecholamine levels and normal adrenal images. The differential diagnosis for a pancreatic mass includes pancreatic carcinoma, lymphoma, cystic neoplasm, and neuroendocrine tumor. All markers of neuroendocrine excess are normal, though elevations can be episodic. The normal 5-HIAA level makes carcinoid syndrome unlikely. VIPomas are associated with flushing, but the absence of profound and protracted diarrhea makes a VIPoma unlikely.

As hypoglycemia from a pancreatic insulinoma is plausible as a cause of episodic loss of consciousness lasting 15 minutes or more, it is important to inquire if giving food or drink helped resolve previous episodes. The normal insulin

\section{TABLE 1. Neuroendocrine Tests}

\begin{tabular}{llc}
\hline Test & Value & Normal Range \\
\hline Serum catecholamine & & \\
\hline Dopamine & $<20$ & $0-20 \mathrm{pg} / \mathrm{mL}$ \\
\hline Norepinephrine & 266 & $80-520 \mathrm{pg} / \mathrm{mL}$ \\
\hline Epinephrine & $<10$ & $10-200 \mathrm{pg} / \mathrm{mL}$ \\
\hline 24-hour urine catecholamine & & \\
\hline Dopamine & 57 & $52-480 \mu \mathrm{g} / 24 \mathrm{~h}$ \\
\hline Norepinephrine & 13 & $15-100 \mu \mathrm{g} / 24 \mathrm{~h}$ \\
\hline Epinephrine & $<6$ & $2-24 \mu \mathrm{g} / 24 \mathrm{~h}$ \\
\hline Chromogranin A & 3.6 & $1.9-15 \mathrm{ng} / \mathrm{mL}$ \\
\hline 24-hour urine 5-HIAA & 4.0 & $0.0-10.0 \mathrm{mg} / 24 \mathrm{~h}$ \\
\hline Insulin & 8.2 & $1-24 \mu \mathrm{ll} / \mathrm{mL}$ \\
\hline Serotonin & 66 & $\leq 230 \mathrm{ng} / \mathrm{mL}$ \\
\hline Vasoactive intestinal polypeptide & 25 & $<75 \mathrm{pg} / \mathrm{mL}$ \\
\hline Somatostatin & 25 & $\leq 30 \mathrm{pg} / \mathrm{mL}$ \\
\hline NOTE: Abbreviation: 5-HIAA, 5-hydroxyindoleacetic acid. & & \\
\hline & & \\
\hline
\end{tabular}


level reported here is of limited value, because it is the combination of insulin and C-peptide levels at time of hypoglycemia that is diagnostic. The normal glucose level recorded during one of the earlier episodes and the hypotension argue against hypoglycemia.

The elevated tryptase level is an indicator of mast cell degranulation. Tryptase levels are transiently elevated during the initial 2 to 4 hours after an anaphylactic episode and then normalize. An elevated level many hours or days later is considered a sign of mast cell excess. Although there is no evidence of the multi-organ disease (eg, cytopenia, bone disease, hepatosplenomegaly) seen in patients with a high systemic burden of mast cells, mast cell disorders exist on a spectrum. There may be a focal excess of mast cells confined to one organ or an isolated mass.

The same day as discharge, the patient's wife drove them to the grocery store. He remained in the car while she shopped. When she returned, she found him confused and minimally responsive with subsequent brief loss of consciousness. He was taken to an ED, where he was flushed and hypotensive (systolic BP, $60 \mathrm{~mm} \mathrm{Hg}$ ) and tachycardic. Other examination findings were normal. After fluid resuscitation he became alert and oriented. WBC count was $20,850 / \mu \mathrm{L}$ with $89 \%$ neutrophils, hemoglobin level was $14.6 \mathrm{~g} / \mathrm{dL}$, and platelet count was $168,000 / \mu \mathrm{L}$. Serum lactate level was $3.7 \mathrm{mmol} / \mathrm{L}$ (normal, <2.3 $\mathrm{mmol} / \mathrm{L}$ ). Chest radiograph was normal. He was treated with broad-spectrum antibiotic therapy and admitted to the hospital. Blood and urine cultures were sterile. Fine-needle aspiration of the pancreatic mass demonstrated nonspecific inflammation. Four days after admission (3 days after pancreatic mass biopsy) the patient developed palpitations, felt unwell, and had marked flushing of the face and trunk, with concomitant $\mathrm{BP}$ of $90 / 50 \mathrm{~mm} \mathrm{Hg}$ and HR of $140 \mathrm{bpm}$.

The salient features of this case are recurrent hypotension, tachycardia, and flushing. Autonomic insufficiency, to which elderly patients are prone, causes hemodynamic perturbations but rarely flushing. The patient does not have diabetes mellitus, Parkinson disease, or another condition that puts him at risk for dysautonomia. Pancreatic neuroendocrine tumors secrete mediators that lead to vasodilation and hypotension but are unlikely given the clinical and biochemical data.

The patient's symptoms are consistent with anaphylaxis, though prototypical immunoglobulin E (IgE)-mediated anaphylaxis is usually accompanied by urticaria, angioedema, and wheezing, which have been absent during his presentations. There are no clear food, pharmacologic, or environmental precipitants.

Recurrent anaphylaxis can be a manifestation of mast cell excess (eg, cutaneous or systemic mastocytosis). A markedly elevated tryptase level during an anaphylactic episode is consistent with mastocytosis or IgE-mediated anaphylaxis. An elevated baseline tryptase level days after an anaphylactic episode signals increased mast cell burden. There may be a reservoir of mast cells in the bone marrow. Alternatively, the hypervascular pancreatic mass may be a mastocytoma or a mast cell sarcoma (missed because of inadequate sampling or staining).

The lactic acidosis likely reflects global tissue hypoperfusion from vasodilatory hypotension. The leukocytosis may reflect WBC mobilization secondary to endogenous corticosteroids and catecholamines in response to hypotension or may be a direct response to the release of mast cell-derived mediators of inflammation.

The patient was treated with diphenhydramine and ranitidine. Serum tryptase level was $46.8 \mathrm{ng} / \mathrm{mL}$ (nor$\mathrm{mal},<11.5 \mathrm{ng} / \mathrm{mL}$ ), and 24-hour urine histamine level was $95 \mu \mathrm{g} / \mathrm{dL}$ (normal, $<60 \mu \mathrm{g} / \mathrm{dL}$ ). Bone marrow biopsy results showed multifocal dense infiltrative aggregates of mast cells ( $>15$ cells/aggregate), which were confirmed by CD117 (Kit) and tryptase positivity (Figure). Mutation analysis for Kit Asp816Val, which is present in $80 \%$ to $90 \%$ of patients with mastocytosis, was positive. He fulfilled the 2008 World Health Organization criteria for systemic mastocytosis (Table 2). Prednisone, histamine inhibitors, and montelukast were prescribed. Six months later, magnetic resonance imaging of the abdomen showed no change in the pancreatic mass, which was now characterized as a possible splenule. The patient had no additional episodes of flushing or syncope over 2 years.


FIG. Bone marrow biopsy histopathology. (A) Dense aggregate of $>15$ abnormal spindled mast cells (arrow); similar aggregates are multifocal throughout biopsy (hematoxylin-eosin stain). Immunohistochemical stains for CD117 (Kit) (B) and tryptase (C) confirm that spindled cells within aggregates are mast cells (arrows). Mast cells also aberrantly express CD2 and CD25 (not shown). 
TABLE 2. World Health Organization Criteria for Diagnosis of Systemic Mastocytosis ${ }^{a}$

Major
- Multifocal dense mast-cell infiltrate (>15 mast cells per infiltrate) in bone marrow and/or
extracutaneous organs
Minor
- Presence of Asp816Val Kit mutation in bone marrow, peripheral blood, or extracutaneous tissue
- Expression of CD117 (Kit) with CD2 and/or CD25 in mast cells of bone marrow
- $>25 \%$ atypical or spindle-shaped mast cells
- Serum tryptase $>20 \mathrm{ng} / \mathrm{mL}$
aDiagnosis requires either 1 major criterion +1 minor criterion or 3 minor criteria.

\section{DISCUSSION}

Cardiovascular collapse (hypotension, tachycardia, syncope) in an elderly patient prompts clinicians to focus on life-threatening conditions, such as acute coronary syndrome, pulmonary embolus, arrhythmia, and sepsis. Each of these diagnoses was considered early in the course of this patient's presentations, but each was deemed unlikely as it became apparent that the episodes were self-limited and recurrent over years. Incorporating flushing into the diagnostic problem representation allowed the clinicians to focus on a subset of causes of hypotension.

Flushing disorders may be classified by whether they are mediated by the autonomic nervous system (wet flushes, because they are usually accompanied by diaphoresis) or by exogenous or endogenous vasoactive substances (dry flushes). Autonomic nervous system flushing is triggered by emotions, fever, exercise, perimenopause (hot flashes), and neurologic conditions (eg, Parkinson disease, spinal cord injury, multiple sclerosis). Vasoactive flushing precipitants include drugs (eg, niacin); alcohol (secondary to cutaneous vasodilation, or acetaldehyde particularly in people with insufficient acetaldehyde dehydrogenase activity ${ }^{2}$; foods that contain capsaicin, tyramine, sulfites, or histamine (eg, eating improperly handled fish can cause scombroid poisoning); and anaphylaxis. Rare causes of vasoactive flushing include carcinoid syndrome, pheochromocytoma, medullary thyroid carcinoma, VIPoma, and mastocytosis. ${ }^{2}$

Mastocytosis is a rare clonal disorder characterized by the accumulation of abnormal mast cells in the skin (cutaneous mastocytosis), in multiple organs (systemic mastocytosis), or in a solid tumor (mastocytoma). Urticaria pigmentosa is the most common form of cutaneous mastocytosis; it is seen more often in children than in adults and typically is associated with a maculopapular rash and dermatographism. Systemic mastocytosis is the most common form of the disorder in adults. ${ }^{3}$ Symptoms are related to mast cell infiltration or mast cell mediator-related effects, which range from itching, flushing, and diarrhea to hypotension and anaphylaxis. Other manifestations are fatigue, urticaria pigmentosa, osteoporosis, hepatosplenomegaly, bone pain, cytopenias, and lymphadenopathy. ${ }^{4}$

Systemic mastocytosis can occur at any age and should be considered in patients with recurrent unexplained flushing, syncope, or hypotension. Eighty percent to $90 \%$ of patients with systemic mastocytosis have a mutation in $\mathrm{Kit}^{,}{ }^{5}$ a transmembrane tyrosine kinase that is the receptor for stem cell factor. The Asp816Val mutation leads to increased proliferation and reduced apoptosis of mast cells. ${ }^{3,6,7}$ Proposed diagnostic algorithms ${ }^{8-11}$ involve measurement of serum tryptase levels and examination of bone marrow. Bone marrow biopsy and testing for the Asp816Val Kit mutation should be considered in patients with modestly elevated baseline tryptase levels $(11.5-20 \mathrm{ng} / \mathrm{mL})$ if clinical findings are consistent with mastocytosis. ${ }^{12}$

The primary goals of treatment are managing mast cellmediated symptoms and, in advanced cases, achieving cytoreduction. Alcohol can trigger mast cell degranulation in indolent systemic mastocytosis and should be avoided. Mast cell-mediated symptoms are managed with histamine blockers, leukotriene antagonists, and mast cell stabilizers. ${ }^{12}$ Targeted therapy with tyrosine kinase inhibitors (eg, imatinib) in patients with transmembrane Kit mutation (eg, Phe522Cys, Lys509Ile) associated with systemic mastocytosis has had promising results. ${ }^{13,14}$ However, this patient's Asp816Val mutation is in the Kit catalytic domain, not the transmembrane region, and therefore would not be expected to respond to imatinib. A recent open-label trial of the multikinase inhibitor midostaurin demonstrated resolution of organ damage, reduced bone marrow burden, and lowered serum tryptase levels in patients with advanced systemic mastocytosis. ${ }^{15}$ Interferon, cladribine, and high-dose corticosteroids are prescribed in patients for whom other therapies have been ineffective. ${ }^{8}$

The differential diagnosis is broad for both hypotension and for flushing, but the differential diagnosis for recurrent hypotension and flushing is limited. Recognizing that flushing was an essential feature of this patient's hypotensive condition, and not an epiphenomenon of syncope, allowed the clinicians to focus on the overlap and make a shocking diagnosis.

\section{Acknowledgment}

The authors thank David Bosler, MD (Cleveland Clinic) for interpreting the pathology image.

Disclosure: Nothing to report.

\section{References}

1. Wilkin JK. The red face: flushing disorders. Clin Dermatol. 1993;11(2):211-223.

2. Izikson L, English JC 3rd, Zirwas MJ. The flushing patient: differential diagnosis, workup, and treatment. J Am Acad Dermatol. 2006;55(2):193-208.

3. Valent P, Akin C, Escribano L, et al. Standards and standardization in mastocytosis: consensus statements on diagnostics, treatment recommendations and response criteria. Eur J Clin Invest. 2007;37(6):435-453.

4. Hermans MA, Rietveld MJ, van Laar JA, et al. Systemic mastocytosis: a cohort study on clinical characteristics of 136 patients in a large tertiary centre. Eur J Intern Med. 2016;30:25-30.

5. Kristensen T, Vestergaard H, Bindslev-Jensen C, Møller MB, Broesby-Olsen S; Mastocytosis Centre, Odense University Hospital (MastOUH). Sensitive KIT D816V mutation analysis of blood as a diagnostic test in mastocytosis. Am J Hematol. 2014;89(5):493-498.

6. Verstovsek S. Advanced systemic mastocytosis: the impact of KIT mutations in diagnosis, treatment, and progression. Eur J Haematol. 2013;90(2):89-98. 
7. Garcia-Montero AC, Jara-Acevedo M, Teodosio C, et al. KIT mutation in mast cells and other bone marrow hematopoietic cell lineages in systemic mast cell disorders: a prospective study of the Spanish Network on Mastocytosis (REMA) in a series of 113 patients. Blood. 2006;108(7):2366-2372.

8. Pardanani A. Systemic mastocytosis in adults: 2015 update on diagnosis, risk stratification, and management. Am J Hematol. 2015;90(3):250-262.

9. Valent P, Aberer E, Beham-Schmid C, et al. Guidelines and diagnostic algorithm for patients with suspected systemic mastocytosis: a proposal of the Austrian Competence Network (AUCNM). Am J Blood Res. 2013;3(2):174-180.

10. Valent P, Escribano L, Broesby-Olsen S, et al; European Competence Network on Mastocytosis. Proposed diagnostic algorithm for patients with suspected mastocytosis: a proposal of the European Competence Network on Mastocytosis. Allergy. 2014;69(10):1267-1274
11. Akin C, Soto D, Brittain E, et al. Tryptase haplotype in mastocytosis: relationship to disease variant and diagnostic utility of total tryptase levels. Clin Immunol. 2007;123(3):268-271.

12. Theoharides TC, Valent P, Akin C. Mast cells, mastocytosis, and related disorders. N Engl J Med. 2015;373(19):1885-1886.

13. Akin C, Fumo G, Yavuz AS, Lipsky PE, Neckers L, Metcalfe DD. A novel form of mastocytosis associated with a transmembrane c-kit mutation and response to imatinib. Blood. 2004;103(8):3222-3225.

14. Zhang LY, Smith ML, Schultheis B, et al. A novel K509I mutation of KIT identified in familial mastocytosis-in vitro and in vivo responsiveness to imatinib therapy. Leuk Res. 2006;30(4):373-378.

15. Gotlib J, Kluin-Nelemans HC, George TI, et al. Efficacy and safety of midostaurin in advanced systemic mastocytosis. N Engl J Med. 2016;374(26):2530-2541. 